\title{
Hiperinsulinismo en recién nacido
}

\author{
Dra. Sylvia Aмелjo M. ${ }^{1}$; Dra. Andrea Gleisner E. ${ }^{1}$; Dra. Vera Wilhelm P. ${ }^{1}$; Dr. Julio Salas C. ${ }^{2}$; \\ Dr. Sergio Treuer P. ${ }^{2}$; Dr. Sergio Rojas C.37 Dr. Francisco Mucientes F. 4
}

\section{Hyperinsulinism in the newborn}

Two newborn infants with scvere hypoglycemia secondary to hyperinsulinism are reported. The excessive insulin secretion was confirmed by radioinmunoassay. Resection of approximately $95 \%$ of the pancreatic tissue was performed in both patients, resulting in adecuate control of their iypoglicemia. Preliminary histological studies of the pancreatic tissties showcd nesidioblastosis with a small component of endocrine dysplasia in one of the cases, and endocrinc dysplasia with a small component of nesidioblastosis in the other.

(Key words: Hypoglicemia, hyperinsulírism, nesidioblastosis, pancreatic endoctine dy splasid, newborm).

La hipoglicemia en el periodo neonatal, sintomática o no, es por lo general transitoria. La hipoglicemia recurrente o persistente es menos frecuente y se caracteriza por prolongarse después de la primera semana de vida y requerir aportes importantes de glucosa endovenosa para mantener la glicemia en rangos normales, constjtllye una urgencia médica y un problema diagnóstico y terapéutico. Sus causas pueden ser las anomalias hereditarias del metabolismo de los hidratos de carbono o de los aminoácidos, los déficit de hormonas de crecimiento, adrenocorticotrofin $y$ cortisol o el hiperinsulinismo. Cualquiera sea la causi de este trastorno metabólico es indispensabie su tratamiento adecuado para prevenir secuelas neurológicas.

Se describen dos casos de hipoglicemia persistente en recién nacidos, secundaria en ambos a hiperinsulinismo, que fueron tratados mediante resección subtotal del tejido pancreatico con buenos resultados.

\section{Casos Clínicos}

1. Niña, nacida el 7 de junio de 1985 , padres consanguíneos. Una hermana de sexo femenino y

1. Endocrinología infantil, departamențo de pediatria. Ltuiversidad de Concepción.

2. Residente becario, departamento de pediatría. Universidad de Concepción.

3. Cirujano infantil, Hospital Leonor Mascayarto, Concepción.

4. Departamento de jatología, Cniversidad de Concepción. peso de nacimiento de $5.500 \mathrm{~g}$ falleció a los 11 días de vida; otro de sexo masculino que pesó al nacer $5.000 \mathrm{~g}$ muriố a los 23 días de vída, ambos en forma súbitat, después de presentar decaimien to y cianosis. Dos hermanas vjvas, una sana y otra en rratamiento por epilepsia. La madre había sufrido, además, un aborto espontáneo. La paciente fue producto del $6^{\circ} \mathrm{embarazo}$, sin complicaciones, nació por cesárea electiva; peso al nacer: $3.700 \mathrm{~g}$; talla: $56 \mathrm{~cm}$; circunferencia craneana: $36 \mathrm{~cm}$. Fue catalogada como recién nacida pretérmino, grande para la edad gestacional (Pr TGEG). 37 semanas, Apgar 9 (1 min) y 10 ( 10 min). A las 4 horas de vida sufrió crisis de cianosis, que coincidió con glicemia de $2 \mathrm{~m} \mathrm{~mol} x$ 1 ( $36 \mathrm{mg} x$ dl) y se inició tratamiento con glucosa endovenosa, pero a las 18 horas de vida tuvo un episodio de apnea y rotación externa de las extremidades, con glicemia de $2,8 \mathrm{~m}$ mol $x \mathrm{l}$ (50 $\mathrm{mg} x \mathrm{dl}$ ) y a las 43 horas de vida estaba asintomática y la glicemia de control era de $1 \mathrm{~m}$ mol x 1 (18 mg $\times$ dl). A las 72 horas de vida fue trasladada a la unidad de recién nacidos del hospital Gmo. Grant Benavente de Concepción para su estudio, requiriendo aportes de glucosa superiores a $12 \mathrm{mg} \mathrm{x} \mathrm{kg} \mathrm{x} \mathrm{min} \mathrm{para} \mathrm{mantener} \mathrm{la}$ glicemia en los rangos normales. La proporción de hemoglobina glicosilada y las pruebas de tolerancia a la glucosa de la madre eran normales. La ecotomografía abdominal fue normal y las mediciones de insulinemia y glicemia, correspondían a hiperinsulinismo (Tabla 1). A los 24 días de vida se le extirpó el $95 \%$ del tejicto pancreático conservando el bazo. Presentó hipesglicemia durante la intervención pero ésta fue controlada mediante una dosis de insulina. Las insulinemias y glicemias realizadas 10 días des. 
Tabla 1.

Paciente 1. Glicemia e insulinemia, antes y después de la pancreatectomía.

\begin{tabular}{|c|c|c|}
\hline $\begin{array}{l}\text { Glicemia* } \\
(\mathrm{m} \operatorname{mol} \times 1)\end{array}$ & $\begin{array}{l}\text { Irtusulinemia } \\
(\mu \cup \mathrm{L} \times \mathrm{ml})\end{array}$ & $\begin{array}{c}\text { Relación l/G } \mathrm{G}^{* *} \\
(\mu \mathrm{U} \times \mathrm{ml} / \mathrm{m} \text { mol } x \mathrm{l})\end{array}$ \\
\hline $\begin{array}{c}\text { Prepancreatectomía } \\
3,5 \\
2,5 \\
3,1\end{array}$ & $\begin{array}{l}12,0 \\
34,0 \\
26,0\end{array}$ & $\begin{array}{r}3,3 \\
13,6 \\
8,4\end{array}$ \\
\hline $\begin{array}{c}\text { Postpancreatectomía } \\
\mathbf{4 , 0} \\
6,5\end{array}$ & $\begin{array}{l}8,8 \\
7,4\end{array}$ & $\begin{array}{l}2,2 \\
1,1\end{array}$ \\
\hline
\end{tabular}

\footnotetext{
$\mathrm{m} \operatorname{mol} \times 1 \times 0,18=y \times 1$

(método de Somogy y Nelson)

** Relación $I / G$ normal: $\leqslant 6$
}

pués de la intervención eran normales (Tabla 1 ).

En el estudio histológico del páncreas se encontró distribución difusa de células alfa y beta en los acinos y mezcla de estas células con las exocrinas; islotes septales muy aislados; hjpertrofia celular en islotes bien formados; nesidioblastosis en conductos intralobulares e interlobulares, con leve predominio de las células alfa sobre las beta en dichas regiones. Se concluyó que habia nesidioblastosis con leve componente de displasia endocrina.

La paciente fue controlada a los 3 meses de vida, mostraba buen desarrollo pondoestatural y sicomotor y en la ecotomografía no fue posible identificar el tejido pancreático.

2. Niña, nacida el 14 de julio de 1985 , 50 hijo de padres sanos no consanguíneos. Una hermana falleció en el periodo de reciěn nacido, de causa no precisada, había pesado al nacer $4.000 \mathrm{~g}$. Otras tres hermanas sanas. La paciente fue producto de un embarazo sin complicaciones, nació por parto vaginal en el hospital Grno. Grant Benavente de Concepción. Sufrió asfixia neonatal por extracción dificil de hombros, Apgar 2 (I min) y 9 (10 min). Peso $4.550 \mathrm{~g}$, talla $56 \mathrm{~cm}$ circunferencia cráneo $36 \mathrm{~cm}$. Fue catalogada como RNTGEG, 39 semanas. A las 3 horas de vida se comprobó hipoglicemia y se trató de acuerdo con el esquema de Cornblath, requiriendo aportes de glucosa superiores a $20 \mathrm{mg} \times \mathrm{kg} \mathrm{x}$ min para mantener la glicemia en rangos aceptables. Se descartó diabetes materna (hemoglobina glicosada normal). La ecotomografia abdominal fue normal. Las mediciones de insulinemia $y$ glicemia confirmaron el diagnóstico de hiperinsulinismo (Tabla 2). A los 25 dias de vida se realizó tratamiento quirúrgico: el páncreas era de tamaño mayor que lo habitual y había tejido pancreático aberrante en la cara anterior de la tercera porción del duodeno. Se realizó la extirpación del $95 \%$ del páncreas y del pancreas aberrante. Durante la intervención presentó hiperglicemia que cedió espontáneamente. La insulinemia y la glicemia medidas diez días después de la operación fueron normales (Tabla 2).

El examen histológico de los tejidos resecados

Tabla 2.

Paciente 2: Glicemin e insulinemia, pre y post pancreatectomía.

\begin{tabular}{|c|c|c|}
\hline $\begin{array}{l}\text { Glicemia } \\
\text { (m mol x 1) }\end{array}$ & $\begin{array}{c}\text { Insulinemia } \\
(\mu \mathrm{L} \times \mathrm{m})\end{array}$ & $\begin{array}{c}\text { Relación } \mathrm{I} / \mathrm{G}^{* *} \\
(\mu \mathrm{U} \times \mathrm{ml} / \mathrm{m} \mathrm{mol} \times 1)\end{array}$ \\
\hline $\begin{array}{c}\text { Prepancreatectomía } \\
2,55 \\
1,05 \\
3,72\end{array}$ & $\begin{array}{r}56,0 \\
100,0 \\
76,0\end{array}$ & $\begin{array}{l}21,9 \\
95,2 \\
20,4\end{array}$ \\
\hline $\begin{array}{l}\text { Postpancreatectomía } \\
5,2\end{array}$ & 6,8 & 1,3 \\
\hline $\begin{array}{ll}\text { * } & \text { mol } \times 1 \times 0,18=\overline{g \times l} \\
& \text { (método de Somogy y Nelson) } \\
\text { ** } \quad \text { Relación IfG normal: }+6\end{array}$ & & \\
\hline
\end{tabular}


reveló mala distribución del componente endocrino, con células alfa y beta entremezcladus con células acinares, islotes mal formados, islotes septales hipertróficos, hipertrofia leve de células endocrinas, leve componente de nesidioblastosis con células alfa y beta en estrecha relación con conductos intralobulares. Conclusión: displasja endocrina con leve componente de nesidioblastosis.

A los tres meses de vida estaba asintomática y tenía buen desarrollo pondoestatural y sicomotor.

\section{DISCUSION}

La hipersecreción de insulina es una causa frecuente de hipoglicemia persistente en recién nacidos. El aspecto de estos pacientes se asemeja al de hijos de madres diabéticas y en ciertas ocasiones el diagnóstico no ofrece mayores dif $\mathrm{i}$ cultades, como-en el caso del síndrome de Beckwith-Wiedemann. En otras oportunidades su reconocimiento no es fácil. pudiendo tratarse de hiperinsulinismos secundarios a hiperplasia de las células beta, adenomas pancreáticos únicos o múltiples, nesidioblastosis o displasia endocrina. En la actualidad no existe una clasificación histológica uniforme en estas afecciones pancreáticas ${ }^{1}$. Laidlaw fue el primero en emplear el término nesidioblastosis en 1938 para referirse a la proliferación đe ișotes de células beta a partir del sistema canalicular pancreático ${ }^{2}$. Estudios recientes han puesto en duda el significado de la nesidioblastosis en recién nacidos y la consideran como una etapa normal del proceso de madura. ción del tejido pancreático, ya que ha sido encontrada en recién racidos normoglicémicos ${ }^{3}$.

Los dos pacientes relatados tienen en común el presentar hipoglicemia desde Jos primeros días de vida la que se prolongó más allá de la primera semana. En ambos las determinaciones plasmáticas de insulina revelan valores elevados tanto absolutos como en relación a la glicemia, siendo la relación insulinemia $\mu \mathrm{Ux} \mathrm{mi} \mathrm{/} \mathrm{glicemia} \mathrm{m} \mathrm{mol}$ $x 1$ superior a seis en todos los $\cos ^{4,5}$. Ante la imposibilidad de instaurar un tratamiento médico con diazóxido por no disponer de este medicamento se procedió a tratamiento quirúrgico precoz, practicándose extirpación de aproximadamente 95\% del páncreas. La extensión de la resección pancreática necesaria para controlar el hiperirtsulinismo es motivo de controversias, pero Ja mayoría de las últimas publicaciones aconsejan resecciones casi totales del páncreas con conservación del bazo, cuando no se logra detectar un insulinoma en el acto quirúrgico ${ }^{1,6,7,8}$. Ambos pacientes presentaron hiperglicemias transitorias en las primeras 24 horas después de la.cirugía: Moazan y cols relatan que uno de cuatro pacientes desarrolló diabetes insulinodependiente y malabsorción con posterioridad a una pancreatectomía de $99 \% 6$; Kramer $y$ cols dicen que aproximadamente la mitad de los pacientes requieren insulina en algún momento de su evolución en forma transitorial. Martin y cols en un seguimiento de 4 meses a 22 años relatan que ningún paciente necesitó insulina ni enzimas pancreáticas. Esto probablemente se debe a la proliferación de los isiotes celulares remanentes ${ }^{8}$.

En el primer caso podría haber un factor gerético causal ya que existe el antecedente de consanguinidad en los padres y de dos hermanos macrosómicos fallecidos en el período de recién nacido de causa no identificada pero compatible con hipoglicemia. La primera publicación de nesidioblastosis familiar fue hecha por woo $y$ cols en 1976, posteriormente han sido descritos otros casos 9.10 . En el segundo paciente no se advierte un componente familiar evidente.

\section{RESUMEN}

Se describen 2 pacientes, recién nacidos, con hipoglicemia secundaria a hiperinsulinemia. Se confirmó la hipersecreción de la hormona mediante radioinmunoanálisis en el plasma. Se extirpó 95\% del tejido pancreático en ambos casos, lográndose el control de la hipoglicemia. Los dos pacientes tuvieron hiperglicemia transitoria en las primeras horas después de la operación. pero diez dias más tarde sus insulinemias $y$ glicemias eran normales y tres meses después mostraban desarrollos pondoestaturales y psico. motrices adecuados. El estudio histológico preliminar reveló nesidioblastosis y leve componente de displasia endocrina en uno de los casos y displasia endocrina con leve componente de nesidioblastosis en el otro.

\section{REFERENCIAS}

1. Kramer, J.L, Bell, M.J., De Schryver, K., Bower, R.J., Ternberg, J.I., White, N.H.: Clinical and histologic indications for Extensive Pancreatic Resection in Nevidioblastosis. An. J. Surg. 143: 116, 1982.

2. Laidkw, G.F.: Nesidioblastoma, the islet tumor of the pancreas. Am. J. Pathol. 14 (2): 125, 1938.

3. Ternberg, J.L.: Surgical treatment of idiopathic hyperinsulinemic hypoglycemia in children. $J$. Thorac. Cardiovasc. Surg. 88: 898, 1984.

4. Linhart, N.. Strouch, Mouhep Leger, L.: Le rapport insulinemic/glycémie. Son intéret diagnostique dans les tumcurs langerhausiennes hypogiycémients. Nuuvelle Presse Med. 3: 1238, 1974.

5. Evrard, A., Berlier, A., Ruilton, A., Francois, R.: Le rapport insulinemic/glycimie. Pediatric. 1: 31 , 1981. 
6. Moazam, F., Rodgers, B.M., Talbert, J.L., Rosen. bloom. A.L.: Near-Total Pancreatectomy in Persistent Infantile Hypoglycemie. Arch. Surg. 117: 1151,1982 .

7. Gruppuso, P.A., de Luca, F., OShea, P.A., Schwartz, R.: Near-Total Pancreatectomy for Hyperinsulinism. Spantaneous remission of resultant diabetes. Acta Paediatr. Scand. 74: 311 , 1985.

8. Martin, L., Ryckman, F.C., Sheldon, C.A.: lixpe- rience with $95 \%$ parcreatectomy and splenjc sacvage for neonatal nesidioblastosis. Am. Surg. 200: 355, 1984.

9. Woo, D., Scopes, J.W., Polak, J.M: Idiopathic Hypoglycamie in sibs with morphological evidence of nesidioblastosis of the pancreas. Arch. Dis. Child. $51: 528,1976$

10. Stanley Schwartz et cols.: Tamilial Nesidioblastosis: Severe Nconatal hypoclycemie in two families. J. Pediatr. 95: 44, 1979. 